\title{
Giant right coronary artery aneurysm with a huge intramural thrombus
}

\author{
Yunis Daralammouri, MD, ${ }^{\mathrm{a}}$ Jörg Fuhrmann, MD, ${ }^{\mathrm{a}}$ and Thomas Kunze, $\mathrm{MD}^{\mathrm{b}}$
}

Coronary artery aneurysm (CAA), first described Bougon in $1812,{ }^{1}$ is a localized dilatation exceeding the diameter of adjacent arterial segments by $50 \% .^{2}$ The uncommon CAAs with diameters larger than $2 \mathrm{~cm}$ are considered giant aneurysms. ${ }^{3,4}$ We report here the case of a giant CAA detected incidentally in a 67-year-old man and successfully resected.

\section{CASE REPORT}

We report the case of a giant CAA in a 67-year-old white man with a history of hypertension who was admitted to our hospital for evaluation of a pericardial cyst detected incidentally during a computed tomographic (CT) scan of his thorax. The patient was clinically free of symptoms. Physical examination revealed inspiratory rales at the base of both lung fields. Electrocardiography showed a normal frequency sinus rhythm; however, initial transthoracic echocardiography revealed a large cystic mass adjacent to the right atrium that was significantly compressed. In addition, we observed nondilated cardiac chambers, a preserved global left ventricular systolic function, and no intracavitary masses (Figure 1, $A)$. The patient was therefore scheduled for surgical resection of the cystic mass. Cardiac catheterization performed before this resection revealed 1 small and 1 giant CAA of

From the Departments of Cardiology ${ }^{\mathrm{a}}$ and Cardiothoracic Surgery, ${ }^{\mathrm{b}}$ Zentralklinik Bad Berka, Bad Berka, Germany.

Disclosures: Authors have nothing to disclose with regard to commercial support.

Received for publication Feb 1, 2013; revisions received Feb 16, 2013; accepted for publication Feb 27, 2013; available ahead of print March 25, 2013.

Address for reprints: Yunis Daralammouri, MD, Cardiology, Zentralklinik Bad Berka, Robert-Koch-Alle 9, Calmettestrasse 3, 99438 Bad Berka, Germany (E-mail: yunis71@yahoo.de)

J Thorac Cardiovasc Surg 2013;146:1290-1

$0022-5223 / \$ 36.00$

Copyright $(c) 2013$ by The American Association for Thoracic Surgery

http://dx.doi.org/10.1016/j.jtcvs.2013.02.065 the right coronary artery (RCA; Figure 1,B). Subsequently, multidetector CT angiography was performed to characterize the aneurysms: the giant CAA was at the proximal part of the RCA $(76 \times 37 \mathrm{~mm})$, and the CAA was at the middle part of the RCA (Figure 2, $A$ and $B$ ). In addition, the giant CAA was lined with a large mural thrombus (Figure 2, $A$ ). The patient underwent successful resection of the giant CAA of the RCA under cardiopulmonary bypass through a median sternotomy. Proximal and distal communications of the RCA were ligated outside the aneurysmal sac; subsequently, coronary artery bypass grafting was performed with a saphenous vein graft to the distal part of the RCA.

\section{DISCUSSION}

Bougon first described CAAs in 1812. ${ }^{1}$ CAAs are defined as localized dilatations that exceed the diameter of adjacent arterial segments by $50 \%{ }^{2}$ Coronary arteries with diameters larger than $2 \mathrm{~cm}$ are defined as giant aneurysms $\mathrm{s}^{3,4}$; these are uncommon lesions with varied clinical presentations. The prevalence of angiographically documented CAAs varies from $0.7 \%$ to $4.9 \%$ among patients who undergo coronary angiography. ${ }^{2}$ The prevalence of giant CAAs is $0.02 \%{ }^{4}$ Most CAAs, as in our case, are asymptomatic. ${ }^{5}$ In most adult cases, CAAs are predominantly secondary to atherosclerotic lesions; however, other causes include autoimmune diseases (eg, Kawasaki disease), vasculitis (eg, Behçet disease), trauma, infection (eg, syphilis), dissection, connective tissue disorders (eg, Ehlers-Danlos syndrome), congenital malformation, drug-eluting stent implantation, and rheumatoid arthritis. $^{2,4}$

Giant CAAs can be detected by noninvasive investigations such as echocardiography, CT, and magnetic resonance imaging, but coronary angiography is required for
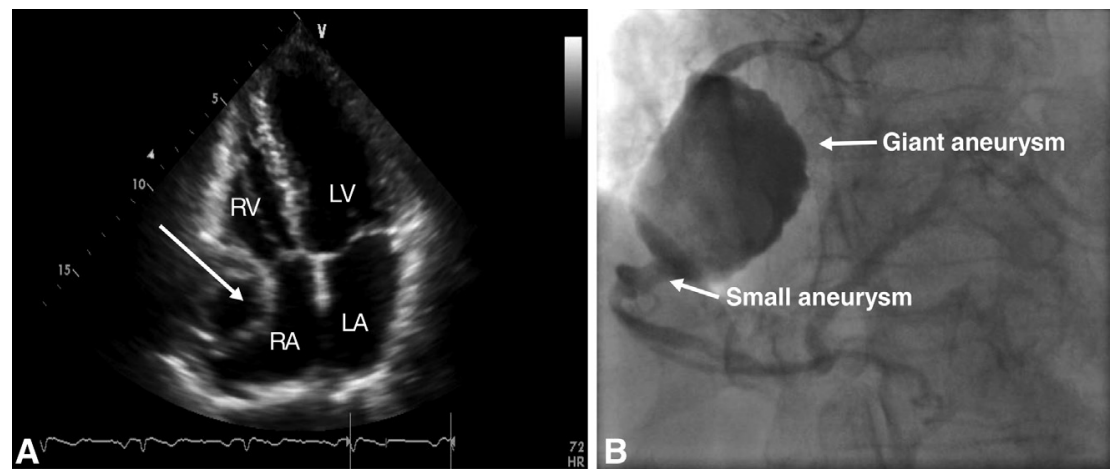

FIGURE 1. A, Echocardiography shows a large cystic mass adjacent to the right atrium $(R A$, arrow). $R V$, Right ventricle; $L V$, left ventricle; $L A$, left atrium. $\mathrm{B}$, Invasive coronary angiography of the right coronary artery demonstrating 2 aneurysms: the giant aneurysm in the proximal part of the right coronary artery and the small aneurysm in the middle part. 


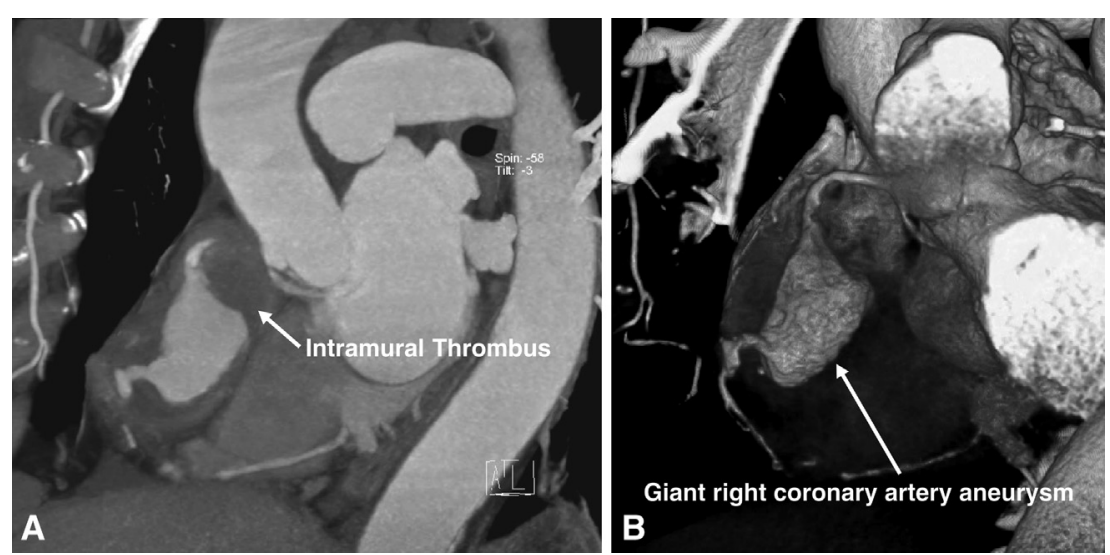

FIGURE 2. A, Axial section computed tomography shows the giant right coronary artery aneurysm (dimensions $76 \times 46 \mathrm{~mm}$ ) with intramural thrombus (arrow). B, In this 3-dimensional heart reconstruction, the 2 right coronary artery aneurysms are clearly visible.

definitive diagnosis and characterization of the aneurysm. In our case, even the coronary angiogram did not show the real size and shape of the giant aneurysm because of the intramural thrombus. In such cases, CT angiography is important for diagnosis and treatment planning.

Thus far there is no optimal management strategy for patients with giant CAAs. For small CAAs, antiplatelet and anticoagulation drugs have been the mainstay of treatment. ${ }^{4}$ Surgical intervention is the usual treatment for giant $\mathrm{CAAs}^{2}$; however, medications (antiplatelet and anticoagulation drugs) and stent implantation have been described. ${ }^{2,4}$

Our patient had an approximately 70-mm giant CAA. Multidetector $\mathrm{CT}$ angiography is important for the diagnosis and treatment of the giant CAA. Surgery and covered stents are potential treatment alternatives. We decided on the former option because of the large intramural thrombus and possible risk of thromboembolism during stent implantation.

\section{References}

1. Jarcho S. Bougon on coronary aneurysm (1812). Am J Cardiol. 1969;24:551-3.

2. Nichols L, Lagana S, Parwani A. Coronary artery aneurysm: a review and hypothesis regarding etiology. Arch Pathol Lab Med. 2008;132:823-8.

3. Jha NK, Ouda HZ, Khan JA, Eising GP, Augustin N. Giant right coronary artery aneurysm-case report and literature review. J Cardiothorac Surg. 2009;4:18.

4. Li D, Wu Q, Sun L, Song Y, Wang W, Pan S, et al. Surgical treatment of giant coronary artery aneurysm. J Thorac Cardiovasc Surg. 2005;130:817-21.

5. Wong CK, Cheng CH, Lau CP, Leung WH. Asymptomatic congenital coronary artery aneurysm in adulthood. Eur Heart J. 1989;10:947-9. 